\title{
Mitigating Shadowing and Topographic Artifacts Using Dual EDS Detectors
}

Shangshang $\mathrm{Mu}^{1}$ and Jens Rafaelsen ${ }^{2}$

${ }^{1}$ EDAX, United States, ${ }^{2}$ Ametek / EDAX LLC, Mahwah, New Jersey, United States

Dual EDS detectors not only double the throughput while maintaining single detector's energy resolution, but also play an important role in mitigating shadowing and topographic artifacts shown in EDS maps of particles or samples with rough or fracture surfaces. For particles or other small objects that are adjacent to large objects, the large objects may create shadow zones for the smaller ones, resulting in X-ray signal attenuation. This effect also applies to samples with severe surface topography that high topography regions may attenuate or block X-ray signals generated from low topography regions. Additional complexities come from surfaces that are significantly inclined towards the detector and other surfaces that are tilted away from the detector, producing take-off angle variations to alter X-ray signal intensities [1]. When the electron beam is scanning over a particle, the take-off angle also changes as the beam moves from one side to the other side of the particle along the spherical surface. With dual detectors, these artifacts associated with shadowing and topography can by compensated for. In the first experiment, dual EDS detectors were positioned with different azimuthal angles to map quartz particles simultaneously and the two sets of data were merged. The Counts Per Second (CPS) maps collected along with elemental maps were used to evaluate topographic and shadowing artifacts. This map type provides a visual representation of the X-ray count rate at every pixel in the dataset, where the brightest pixels represent areas with the highest count rates, while dark and black areas indicate signal attenuation where little to no X-rays are received by the detector or areas with lower X-ray yield. The traditional way to merge map data is to sum the two sets of maps together. However, simply summing does not work quite well on this sample with severe surface topography and shadowing. In the CPS map collected by detector 1 (Figure 1a), the circled dark region shows where X-ray signals are shadowed by adjacent particles, while the corresponding region in the map from detector 2 (Figure 1b) is facing towards the detector to receive more X-ray counts. Adding these two maps together increases the number of counts per pixel, but also gives the shadow artifacts from both detectors (Figure 1c). In Figure 1d, the maximum signal at each pixel between the two single detector CPS maps was used, and the double shadowing and contributions of topography are significantly alleviated. Since the signal from only one map or the other is used, this does not increase statistics or spectral quality. When doing contamination assessment or failure analysis, it is important to cover as many objects of interest as possible. An iron oxide sample was mapped using the same dual EDS detector setup to evaluate the distribution of phosphorus as surface contaminants. The CPS maps from both detectors indicate that this sample has an extremely rough fracture surface with obvious shadow zones (Figure $2 \mathrm{a}$ and $2 \mathrm{~b}$ ). Although their corresponding P K maps show a large amount of phosphorus particles and the distributions look somewhat even (Figure $2 \mathrm{~d}$ and $2 \mathrm{e}$ ), there are missing particles in the circled regions from either elemental map. The topographic effects in the single detector CPS maps are mitigated in the merged CPS map using the maximum signal function (Figure 2c), and the shadowing is resolved in the maximum signal P K map to represent more accurate data (Figure 2f). To quantify the improvement of data quality, the three CPS maps were converted into binary images to measure the density of phosphorus particles. The particle density calculated from maps collected by detector 1 and detector 2 are 6504 particles/mm2 and 6178 particles/mm2, respectively. However, the density in the merged map is 7795 particles/mm2, which means either detector did not capture a significant 
number of particles on the sample surface. Dual EDS detectors are very useful for mitigating the shadowing and topographic effects associated with particles and rough or facture surfaces. Selecting the maximum signals between the two sets of maps from dual detectors is a more efficient way to resolve these artifacts than using the sum of the maps.
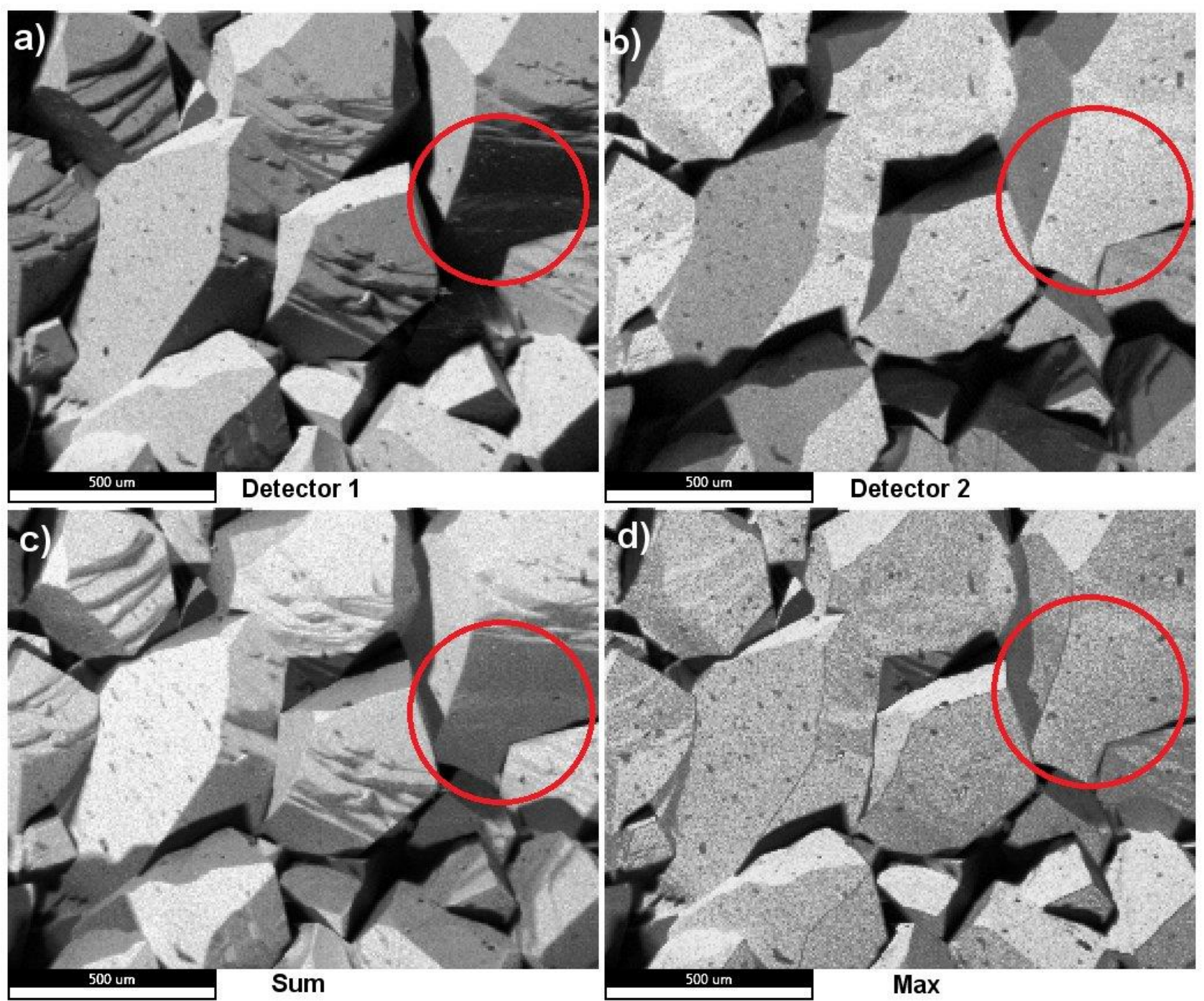

Figure 1. Figure 1. CPS maps of quartz particles from detector 1 (a) and detector 2 (b), sum of the CPS maps from both detectors (c), and maximum signal CPS map (d). 

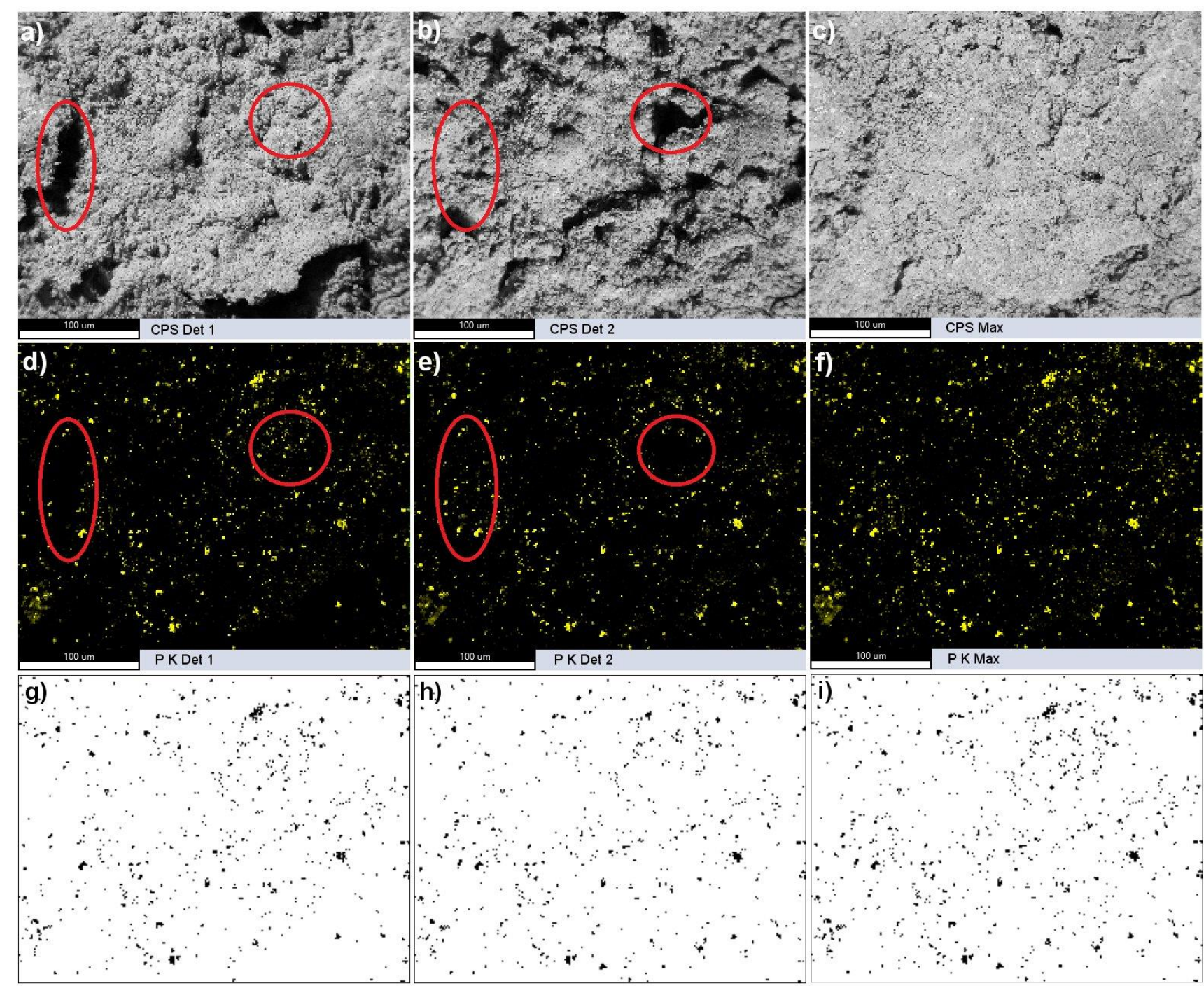

Figure 2. Figure 2. CPS maps collected from detector 1 (a) and detector 2 (b), and maximum signal CPS map (c). (d)-(f): corresponding elemental maps of $\mathrm{P} \mathrm{K}$ of the above maps. The red circles highlight regions with missing signals from either detector. (g)-(i): binary images of the above elemental maps.

References

[1] T. Ronnhult, B. Brox and G. Fritze, "The Influence of Surface Topography on the X-ray Intensity in Electron Microprobe Analysis (EDS/WDS),” Scanning, vol.9, pp. 81-87, 1987. 